\title{
Quality Assessment of Traversability Maps from Aerial LIDAR Data for an Unmanned Ground Vehicle
}

\author{
Nicolas Vandapel, Raghavendra R. Donamukkala and Martial Hebert \\ Carnegie Mellon University \\ 5000 Forbes Avenue \\ Pittsburgh, PA 15213 \\ USA
}

\begin{abstract}
In this paper we address the problem of assessing quantitatively the quality of traversability maps computed from data collected by an airborne laser range finder. Such data is used to plan paths for an unmanned ground vehicle (UGV) prior to the execution of long range traverses. Little attention has been devoted to the problem we address in this paper. We use a unique data set of geodetic control points, real robot navigation data, ground LIDAR (LIght Detection And Ranging) data and aerial imagery, collected during a week long demonstration to support our work.
\end{abstract}

\section{INTRODUCTION}

Numerous results and extensive field tests have been reported on autonomous mobile robot navigation for planetary exploration [15], [10], cross country navigation [3] or polar exploration [11]. Typically the robot performance evaluation is based on successful completion of test missions. We argue that a more pointed evaluation of performance is appropriate to improve the system performance. For example path planning relies on a ground surface model of the terrain whose quality will affect the traversability map and the paths produced. Knowledge about the quality of the traversability map is critical. But few experiments have focused on such topic because of the multi-dimensionality of the problem, the representativity of the terrain to be used, the collection of ground truth and recording of vehicle states that require substantial logistics.

In this paper we address the problem of assessing quantitatively the quality of traversability maps computed a priori from aerial LIDAR data collected over vegetated terrains.

Our problem is an instance of the broader problem of autonomous mobile robot performance evaluation. This difficult problem has recently received increasing attention with the Performance Evaluation of Intelligent System Workshops (PERMIS) organized by NIST [12]. Robotic competitions, like the AAAI [14] or the robocup [5] competition, are common ways to evaluate robot performance. Extensive field test is the other common way as with FIDO in preparation for Mars exploration [15]. We tackle our problem by looking at the quality of the elevation map recovered from raw aerial data, the influence of the vehicle model and by comparing the a priori data analysis and the experimental data collected when the robot traverses a terrain. Our analysis is supported by extensive field tests and data collection.

During the DARPA PerceptOR program [7] our team, lead by General Dynamic Robotic Systems, conducted four one-week-long experiments in various terrains (mountainous, desert, southern meadows, northern forest). During each experiment, long range autonomous navigation was performed by an All Terrain Vehicle (ATV) using a priori aerial LIDAR data for global path planning as reported in [16]. For this paper we use the data collected during the latest experiment we conducted in November 2002 which include aerial LIDAR data, high-resolution geo-referenced aerial imagery, geodetic control points, ground LIDAR data and terrains surveys.

The rest of this article is divided into three sections. Section 2 presents the method used to generate the traversability maps and to plan the paths a priori using aerial LIDAR data. Section 3 describes the assessment of the traversability maps and presents results from extensive field tests. Finally we conclude in Section 4.

\section{Global Path Planning Using Aerial LiDAR DATA}

In this section we report briefly the method used to compute the traversability map from aerial LIDAR data and to produce the paths. The steps described in the next paragraph are illustrated in Figure 1. Details on the path planning method can be found in [16], while details on the vegetation filtering method can be found in [9].

Using raw three-dimensional (3-D) points collected by an aerial LIDAR, the goal is to produce a safe path connecting a set of given intermediate waypoints. The procedure is decomposed into 3 steps: 1) each 3 -D point is classified as ground or vegetation ; 2) a vehicle model and the previous data are used to quantify the risk of traversing each cell along eight specific directions and 3) a path, minimizing the cumulated risk for traversing the cost maps between each waypoint, is finally produced.

Vegetation filtering is of utmost importance because we need to recover, from the raw data, the part of the terrain 


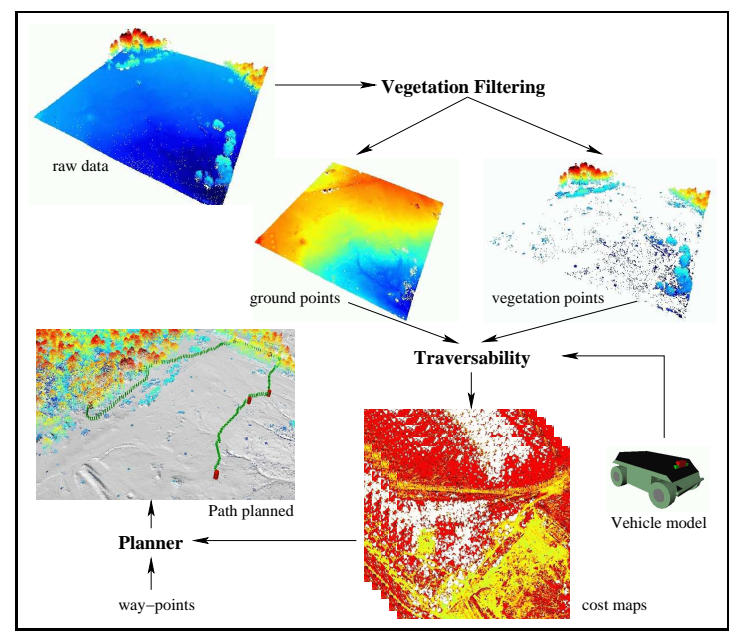

Fig. 1. Problem overview.

the robot will be driving on. In earlier attempts, the vegetation was filtered using the first-last pulse information to initialize a k-mean filter. A second method, based on 3-D convolution, was implemented and produced better results. It is based on the simple fact that the ground points will have no LIDAR return below them. This method can be easily instantiated using a visibility cone. This method is sensitive to the ground terrain slope, the point density and the LIDAR data quality. In the case of sudden changes in elevation, like along a ledge, ground points can be misclassified as vegetation. This can be compensated by dynamically varying the opening of the cone. Isolated canopy points tend to be misclassified as ground data. LIDAR errors can be compensated partially by using a blind window of several decimeters below the point to be classified even though this approach will misclassify vegetation as ground. Results on a variety of terrains are presented in [16], including terrain with steep slopes.

Eight traversability maps are computed by convolving the ground data points with the vehicle model along eight discrete directions. The Northing-Easting plane is discretized into square grid cells, typical 0.5 to $1.0 \mathrm{~m} \mathrm{a}$ side. At each grid cell, a plane is fitted to the locations of the four robot tires. The cost at the cell is a function of the pitch and roll angles of the plane and the ground clearance of the robot. Another map, called the vegetation map, which measures the vegetation clutter at each cell is evaluated. This measure is simply the ratio of the number of points classified (in each grid cell) as vegetation to the total number of points in that cell. This reflects how vegetated the area is. Classical approaches for robot path planning in rough terrain can be found in [8] and [6].

The traversability maps and the vegetation map, along with intermediate waypoints, are input to a Vehicle Level Planner (VLP) to produce an optimum path [2]. The software for path planning was provided by NIST.

\section{EXPERIMENTAL EVALUATION}

In this section we first describe the field test conducted in November 2002. We then present results on surface terrain reconstruction, on the influence of the vehicle model, on map evaluation using actual robot trajectories and using inertial measurements.

\section{A. Field tests}

1) Terrains: The fourth field experiment of our research program, E4, was conducted at Ft Polk, Louisiana, in November 2002. The test range covers an area of one square kilometer and it is traversed by several dirt roads and shallow water streams. The vegetation includes pines, oaks, one meter tall sparce grass and $3 \mathrm{~m}$ tall grass in wet areas. The terrain does not contain any steep slope. The test range is divided into five courses with specific terrain and vegetation characteristics (trees, meadow,water ponds) and man-made obstacles (concertina wires, foxholes, trenches, fort, bunker). Table I, contains the terrain features and obstacles encountered for each course.

TABLE I

TERRAINS CHARACTERISTICS AND FEATURES FOR EACH COURSE

\begin{tabular}{l|l|l} 
Course & Terrain & Obstacles \\
\hline terrain No. 1 & open, trails & bunker \\
\hline terrain No. 2 & $\begin{array}{l}\text { grass, sparse } \\
\text { small trees }\end{array}$ & $\begin{array}{l}\text { concertina wires, fort, } \\
\text { fox-hole }\end{array}$ \\
\hline terrain No. 3 & $\begin{array}{l}\text { trail, woods, wa- } \\
\text { ter streams }\end{array}$ & \\
\hline terrain No. 4 & woods & \\
\hline terrain No. 5 & woods & fort
\end{tabular}

2) Aerial LIDAR Data: The aerial LIDAR data was collected from a manned helicopter equipped with a Saab TopEye mapping system [4]. The operational altitude of the system is $400 \mathrm{~m}$ above the ground level and the laser recovers the first and last pulse, with a $1.8 \mathrm{~m}$ power of separation. The point density varies typically from 4 to 10 points per square meter.

3) Ground truth: Several antennas were installed in order to relay the robot status and navigation data to the base camp. After each course test the terrain was scouted and various pieces of information were collected (DGPS position of salient terrain features or trees, measurement of vegetation height and so forth).

\section{B. Surface terrain reconstruction evaluation}

Accurate recovery of the terrain surface is critical. It depends on the raw 3-D data collected by the LIDAR and on the vegetation filtering technique. We assess both here.

The LIDAR absolute error was estimated using ground features recognizable in the LIDAR data and for which we collected DGPS measurement. We choose three of them: the center of the flat roof of an isolated building, a large 
flat concrete bed and the center of a crater. The results of the first case are presented in table II.

TABLE II

AERIAL LIDAR DATA ABSOLUTE ERROR.

\begin{tabular}{l|r|r|r} 
& DGPS & LIDAR & Error / STD \\
\hline Easting & $485,262.94$ & $485,263.02$ & $0.08 /^{*}$ \\
Northing & $3,437,988.76$ & $3,437,989.01$ & $0.25 /^{*}$ \\
Elevation & 107.00 & 106.90 & $0.1 / 0.0532$
\end{tabular}

For the second feature, the large concrete bed laying at the ground level, the mean elevation error is $4 \mathrm{~cm}$ with a standard deviation of $5.36 \mathrm{~cm}$. For the third feature, the bottom of a large conical hole, the elevation error is $11 \mathrm{~cm}$ and and the horizontal error position is $7 \mathrm{~cm}$. Those results are much better than the accuracy reported in the literature for such a mapping system [4]. But in all cases, the measured elevation of a flat surface fits in a bounding box of $22 \mathrm{~cm}$ height within which we cannot classify points as ground or vegetation. Overall we were able to detect trenches, terrain depression with water, tall grass areas and trails below the tree canopy. But we failed to detect concertina wires, poles and fallen tree trunks below the tree canopy like in the case illustrated in Figure 2.

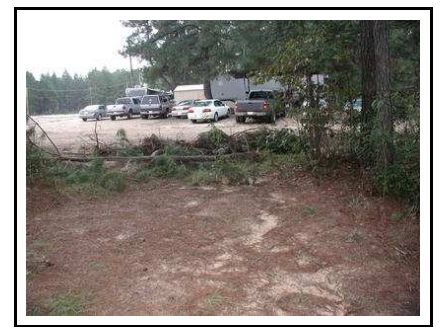

Fig. 2. Example of feature missed, terrain No. 1

In addition, we took a closer look at the quality of the vegetation filtering. We chose a worst case scenario with low point density (one over-fly of the area), with tall brush and thin trees. Figure 3 is a side view of the terrain with in red (blue) the point classified as ground (vegetation). The results obtained are the following: 928 (84) points have been classified as ground (vegetation), 66 vegetation points have been misclassified as ground (7\% of the total), no ground point has been misclassified outside the $22 \mathrm{~cm}$ envelop. The nature of a 3-D point inside that envelop cannot be determined because of the limits on the sensor resolution. This example is representative of the current limitation of the terrain recovery method used.

\section{Influence of the vehicle model}

We used a static vehicle model composed of three parameters: the ground clearance, the traversal and the longitudinal maximum slope angle. We are interested in determining their influence on the path planned. The actual vehicle ground clearance is $17 \mathrm{~cm}$, smaller than the error

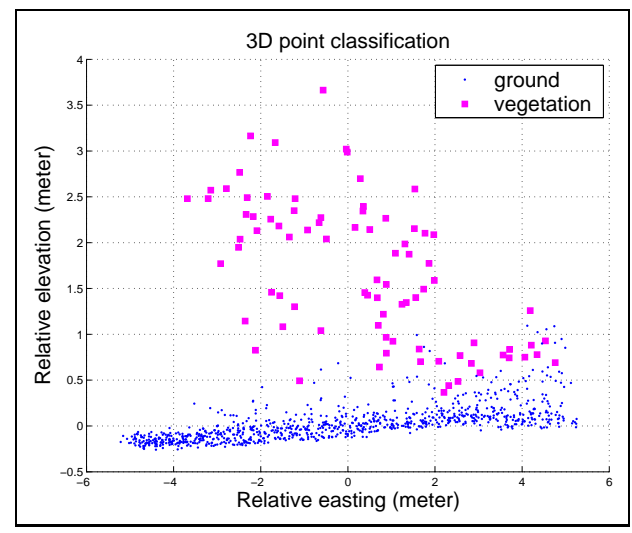

Fig. 3. Vegetation fi ltering error in Area No. 1. Side view of the terrain with in red-square/blue-circle the points classifi ed as vegetation/ground.

envelop of the vegetation filtering. Figure 4 illustrates the influence of this parameter. The example is from the Area No. 2. The terrain is made of tall grass cluttered with trees. A dirt road traverses the terrain. Figure 4-(a) shows the path planned with the actual ground clearance: the trajectory follows the edge of the wood, reach and follow the road and enter the fort. Figure 4-(b) shows a more aggressive path were the ground clearance is set at $50 \mathrm{~cm}$. This is the actual trajectory followed by the robot using the mobility LADAR for obstacle detection. The ground clearance criterion is more sensitive to terrain recovery errors than the maximum slope angles criteria.

\section{Path evaluation using the actual robot trajectory}

In this section we compare the actual paths executed by the robot with the initial path computed using the cost maps. Figure 5 shows such an example for Area No. 1.

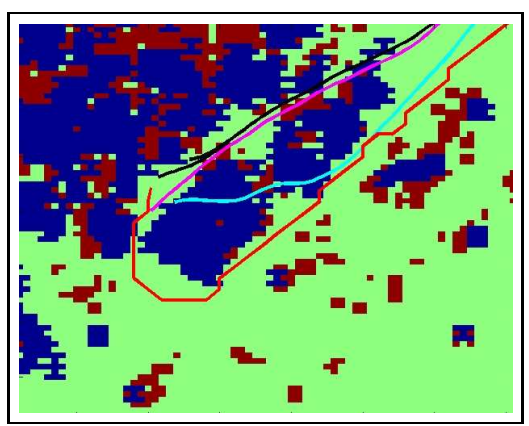

Fig. 5. Actual paths (top) and planned path (bottom, red) overlaid on top of the cost map, terrain No. 1 . The map covers $100 \times 150 \mathrm{~m}$, the grid resolution is $1 \mathrm{~m}$. Color map: In blue/red/green the unknown/nontraversable/other cells

Our criteria would be respectively the path length of the robot in the unknown ${ }^{1}$ and in the non-traversable areas. Table III contains such metrics for the 29 runs performed on the 5 test areas. The first criterion is an indicator of the

\footnotetext{
${ }^{1}$ unknown areas are those where we do not have suffi cient ground hits
} 


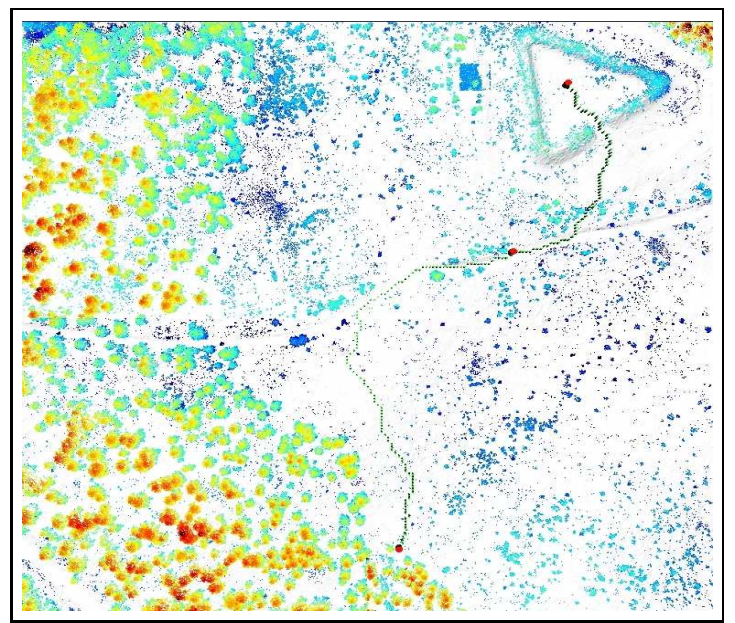

(a) Conservative path (3-D rendering)

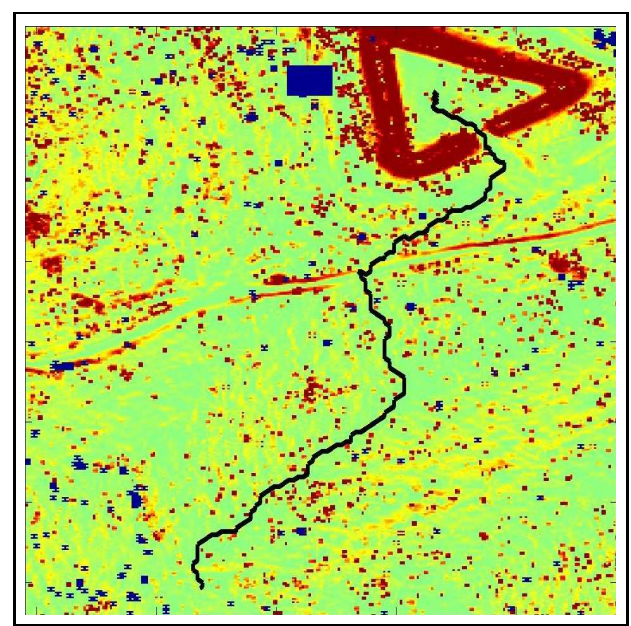

(b) Aggressive path (cost map)

Fig. 4. Ground clearance inflence, terrain No. 2. Figure (a) is a top view of a 3-D rendering of the scene with in white the ground terrain surface represented with a mesh, in color (elevation: blue (low) to red (high)) the 3-D points classifi ed as vegetation. The red poles represents the starting and ending point. The green poles are the intermediate waypoints computed by the path planner. Figure (b) presents the path (black) overlaid on top of a cost map with from green to red the terrain classifi ed as traversable to non-traversable, and in blue the unknown areas.

amount of information missed in areas actually traversed by the robot. This information could be used, given this type of terrain and the neighboring area, to predict the traversability cost in similar regions. The second criterion indicates how conservative we have been or how bad is the terrain surface recovery. This is a partial result dealing only with false-negative cells. The evaluation of false-positive traversable cells requires scouting the site with the robot and collecting ground measurements. Time constraints did not permit doing it. From table III we see that in wooded terrains, area No. 3 and area No. 4, our traversability map evaluation did not perform well as one can expect because of sparse laser beams hitting the ground below the canopy. One can notice that the test areas were not traversed the same number of times. This is only the result of choices made by the field test director during the demonstration.

\section{E. Path evaluation using the robot navigation system}

In this section we propose to compare the vehicle attitude collected during one of the runs with the attitude for the same position computed using the aerial LIDAR data and the ground LIDAR data. Results are presented in Figure 6. The robot drove forward 30 meters, stopped then drove backward 7 meters and finally forward another 10 meters. The robot position was sampled at $20 \mathrm{~Hz}$ and the data set corresponds to 60 seconds of traverse. Figure 6-(d) shows the trajectory of the robot. The terrain is covered by tall grass and bushes. Figure 6-(c) is a top view of the terrain with in grey the ground and in color the vegetation. Figure 6-(a)/(b) presents respectively the pitch and roll of the robot, computed from aerial LIDAR data and from the ground mobility LIDAR data. In the last
TABLE III

TRAVERSES STATISTICS. SHORTER LENGTHS CORRESPOND TO PARTIAL TEST WHERE THE ROBOT WAS DRIVEN IN A SPECIFIC AREA OF INTEREST.

\begin{tabular}{c|c|c|c|c} 
Course & $\begin{array}{c}\text { length } \\
(\mathrm{m})\end{array}$ & $\begin{array}{c}\text { non-trav. } \\
(\mathrm{m})\end{array}$ & $\begin{array}{c}\text { unknown } \\
(\mathrm{m})\end{array}$ & $\%$ \\
\hline & 523.393 & 27.807 & 30.590 & 11 \\
& 223.527 & 5.389 & 32.149 & 16 \\
Area No. 1 & 511.968 & 27.997 & 39.669 & 13.23 \\
& 453.542 & 7.790 & 28.353 & 7.94 \\
& 94.546 & 11.030 & 13.565 & 26.15 \\
& 71.367 & 0.000 & 0.000 & $*$ \\
\hline & 540.793 & 68.226 & 6.772 & 13.87 \\
& 418.814 & 27.821 & 1.186 & 6.93 \\
& 529.215 & 10.897 & 0.000 & 2.02 \\
& 493.695 & 16.896 & 2.828 & 3.99 \\
Area No. 2 & 377.713 & 37.457 & 3.097 & 10.73 \\
& 488.674 & 24.757 & 0.692 & 5.20 \\
& 44.960 & 2.482 & 0.000 & 5.51 \\
& 270.221 & 23.627 & 1.286 & 9.20 \\
& 269.182 & 12.028 & 0.512 & 4.65 \\
& 252.765 & 18.332 & 1.615 & 9.21 \\
\hline & 756.011 & 161.194 & 200.398 & 47.82 \\
Area No. 3 & 671.741 & 120.585 & 167.636 & 42.90 \\
& 640.142 & 79.886 & 279.800 & 56.18 \\
& 584.500 & 111.769 & 170.999 & 48.37 \\
\hline & 218.050 & 18.771 & 71.572 & 41.43 \\
& 198.156 & 33.115 & 51.752 & 42.82 \\
Area No. 4 & 792.302 & 165.352 & 93.857 & 32.71 \\
& 678.827 & 82.980 & 57.225 & 20.65 \\
& 636.430 & 101.558 & 38.451 & 21.99 \\
& 692.529 & 78.274 & 65.696 & 20.78 \\
\hline & 282.721 & 28.560 & 23.953 & 18.57 \\
Area No. 5 & 215.284 & 23.953 & 22.491 & 21.57 \\
& 450.338 & 49.702 & 15.420 & 14.46 \\
& & & &
\end{tabular}

two cases the DGPS ground truth of the robot position and the heading from the inertial navigation system was used to convolve a model of the robot with the LIDAR 
data. From Figure 6, we can see that the frequencies of the curves are the same but the amplitudes are different. Amplitude differences at regime transitions, around frame No. 400 for example, are probably due to the static model of the vehicle we are using. Amplitude differences at static regimes are probably due to the sensor error and terrain recovery process. In either case, in that particular example at least, our traversability map computation has been able to recover the basic trend of the robot attitude. Such analysis could be used to fine tune the cost map generation so as to match actual robot characteristics.

\section{CONCLUSION}

In this article we investigated several methods to evaluate quantitatively the fidelity of traversability maps produced from aerial LIDAR data for autonomous ground mobile robot navigation. We evaluated the surface terrain reconstruction error, we tested the influence of the vehicle model, we produced an estimate of how well we reconstructed the terrain based on real path data and the cost map computed, finally we compared the robot attitude with the estimated robot attitude from aerial and ground LIDAR data.

This work has been possible because of a unique data set. But traversability maps are manually engineered (algorithms, vehicle model threshold) and cannot capture completely the complexity of the terrain. We tried to overcome this problem by using data from actuals runs. We plan to pursue the use of actual robot data to assess the traversability map quality.

\section{ACKNOWLEDGMENTS}

This project was supported by DARPA under the PerceptOR program, under subcontract to General Dynamics Robotic Systems. This work would not have been possible without the help of W. Klarquist and Jeremy Nett from PercepTEK. A special thank to Mike Perschbacher for his help with the DGPS.

\section{REFERENCES}

[1] R. Arvidson, S. Squyres, E. Baumgartner, L. Dorsky, P. Schenker, "Rover trials for Mars sample return mission prove successful", EOS Transactions, American Geophysical Union, vol. 81, no. 7, February 2000, pp. 65-72.

[2] S. Balakirsky, A. Lacaze, "World modeling and behavior generation for autonomous ground vehicle", in Proceedings of 2000 IEEE International Conference on Robotics and Automation, pp. 1201-1206, vol. 2.

[3] P. Bellutta, R. Manduchi, L. Matthies, K. Owens, A. Rankin, "Terrain perception for DEMO III", in Proceedings of 2000 IEEE Intelligent Vehicles Symposium, pp. 326-331.
[4] E.P. Baltsavias, Airborne laser scanning: existing systems and firms and other resources, ISPR Journal Journal of Photogrammetry \& Remote Sensing, vol. 54, no. 2-3, July 1999, pp. 199-214.

[5] H. Burkhard, D. Duhaut, M. Fujita, P. Lima, R. Murphy, R. Rojas, "The road to RoboCup 2050", IEEE Robotics \& Automation Magazine, vol. 9, no. 2, june 2002, pp. 31-38.

[6] M. Cherif, "Motion planning for All-Terrain Vehicles: a physical modeling approach for coping with dydnamic and contact interaction constraints", IEEE Transaction on Robotics and Automation, vol. 15, no. 2, April 1999, pp. 202-218.

[7] S. Fish, "Overview of UGCV and PerceptOR status", in Proceedings of 2003 Unmanned Ground Vehicle Technology $V$, vol. 5083.

[8] A. Hail, T. Simeon, M. Taix, "Algorithms for rough terrain trajectory planning" Advanced Robotics, vol. 16, no. 8, 2002, pp.673-699.

[9] M. Hebert, N. Vandapel, S. Keller, R.R. Donamukkala, "Evaluation and comparison of terrain classification techniques from LADAR data for autonomous navigation", in Proceedings of 2002 Army Science Conference.

[10] S. Lacroix, A. Mallet, D. Bonnafous, G. Bauzil, S. Fleury, M. Herrb, R. Chatila, "Autonomous rover navigation on unknown terrains: functions and integration", Internatioal Journal of Robotics Research, vol. 21, no.10, October 2002, pp. 917-942.

[11] S. Moorehead, R. Simmons, D. Apostolopoulos, W. Whittaker, "Autonomous navigation field results of a planetary analog robot in Antarctica", in Proceedings 1999 International Symposium on Artificial Intelligence, Robotics and Automation in Space, pp.237-242.

[12] E. Messina and A. Meystel, Proceedings of performance metrics for intelligent systems workshop, NIST, 2002.

[13] T. Stentz, A. Kelly, H. Herman, P. Rander, Mandelbaum R., "New results for integrated air/ground vehicle system for semi-Autonomous off-road Navigation", in Proceedings of 2003 AUVSI Unmanned Systems.

[14] W. Smart, T. Balch, H. Yanco, AAAI Workshop on Mobile Robot Competition and Exhibition, AAAI, 2002.

[15] E. Tunstel et al.,"'FIDO rover field trials as rehearsal for the NASA 2003 Mars exploration rovers mission", in Proceedings 2002 World Automation Congress, International Symposium on Robotics with Applications, pp. 320-327.

[16] N. Vandapel, R. Donamukkala, M. Hebert, "Experimental results in using aerial LIDAR data for mobile robot navigation, in Proceedings 2003 International Conference on Field and Service Robotics. 


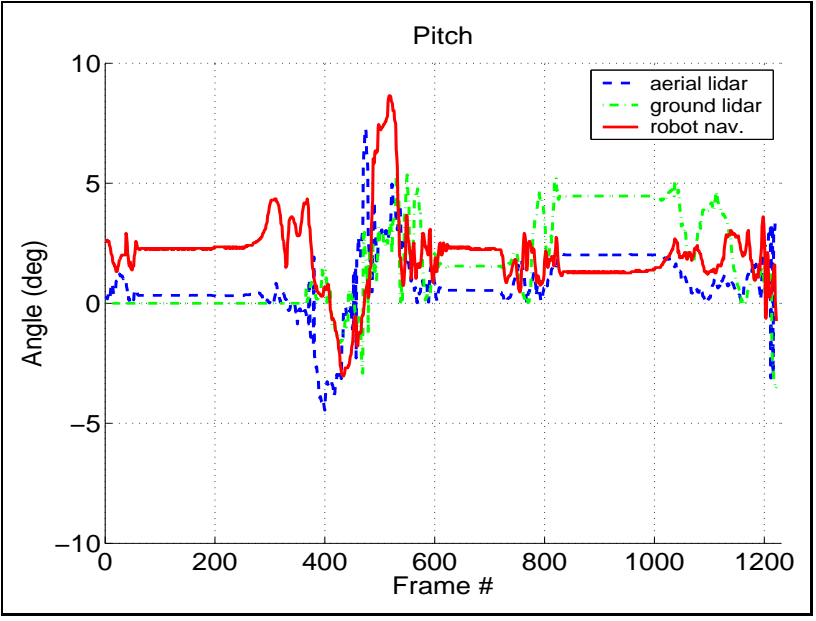

(a) Pitch

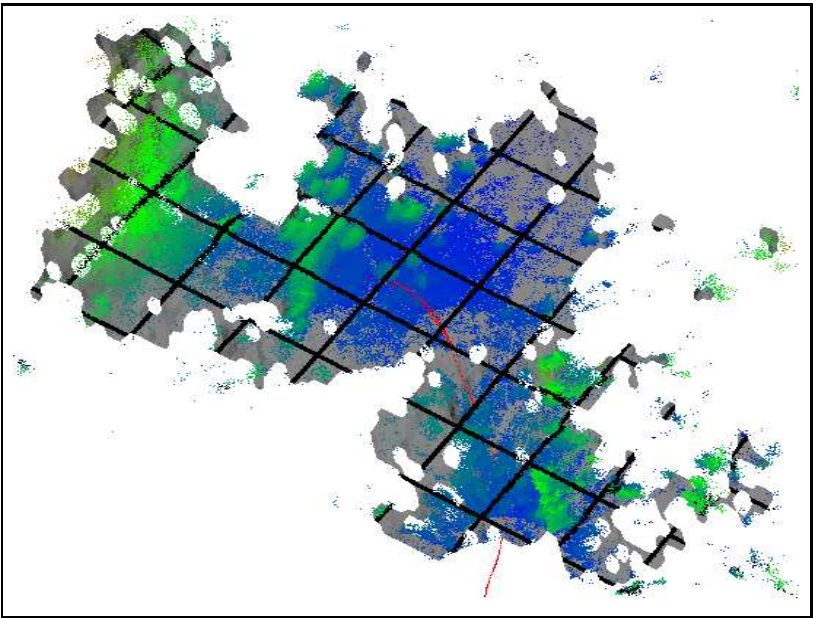

(c) Top view terrain

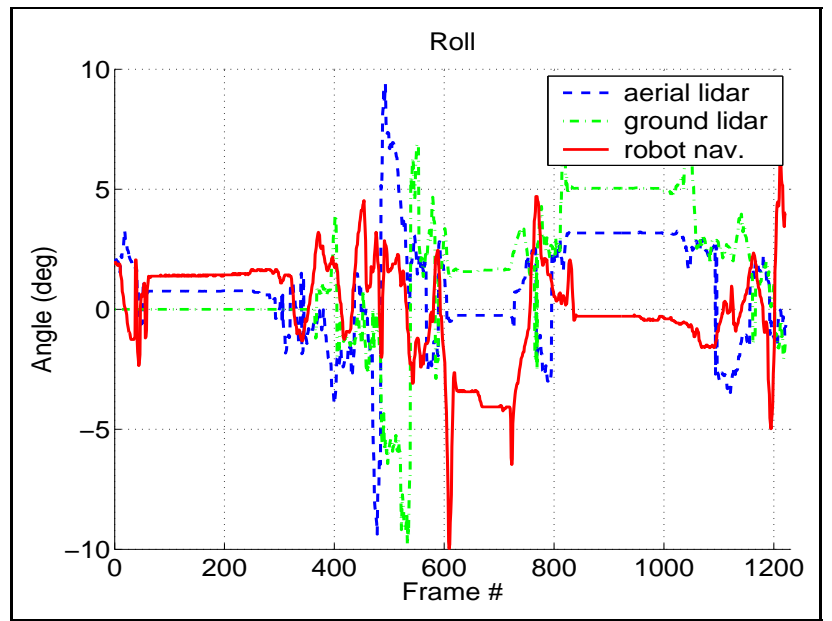

(b) Roll

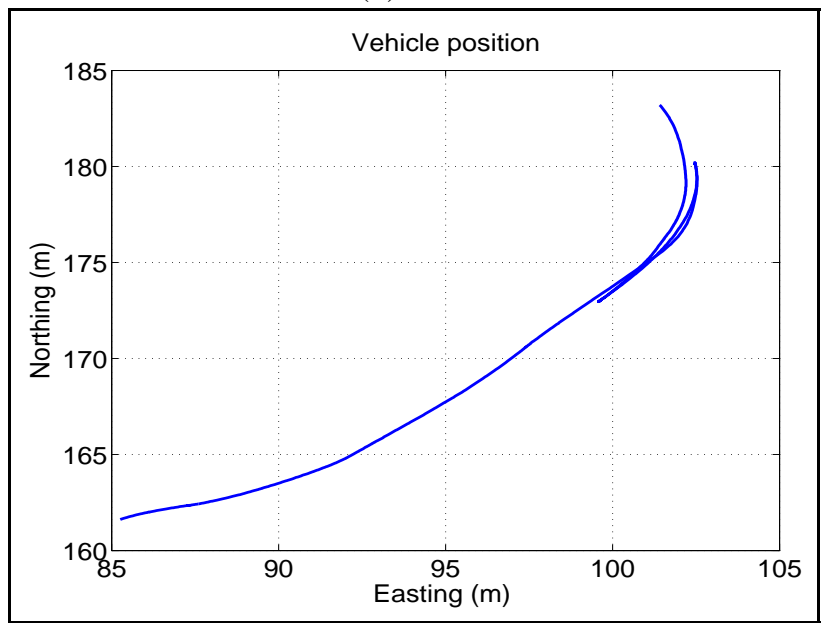

(d) Vehicle trajectory

Fig. 6. (a-b) Vehicle attitude: red line from robot navigation data, blue dashed line from aerial LIDAR data, green dash-dot line from ground LIDAR data. (c) Terrain: in grey, surface terrain $0.5 \mathrm{~m}$ resolution, black grid spaced by $2 \mathrm{~m}$, in red the robot trajectory and in color (from blue to green) the vegetation with the elevation color coded. Results are from Area No. 2

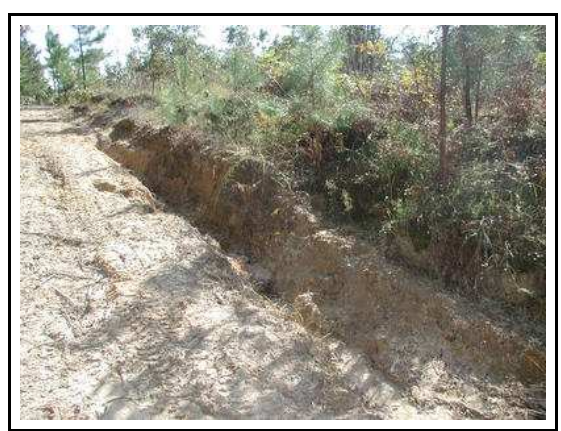

(a) Scene picture

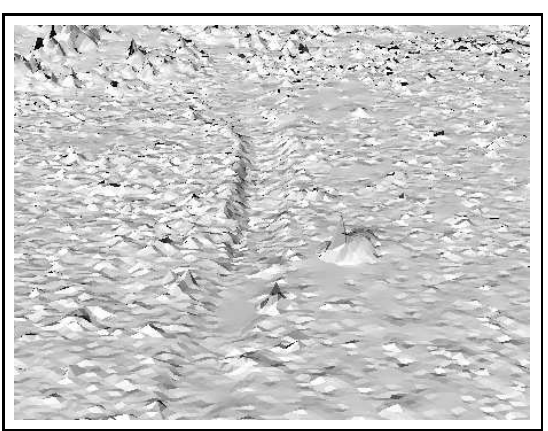

(b) 3-D terrain model (vegetation filtered)

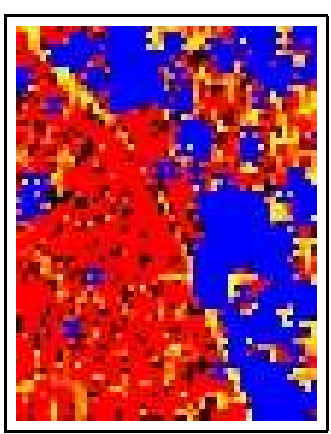

(c) Cost map

Fig. 7. Trench detection, Area No. 3. Color map: in blue the unknown area, for the other cells, from green to red, traversable to non-traversable 